\title{
Randomized Study on the Effectiveness of \\ Nomegestrol Acetate Plus 17 $\beta$-Estradiol Oral \\ Contraceptive vs Dienogest Oral Pill in Women with Endometriosis-Associated Chronic Pelvic Pain
}

\section{Salvatore Caruso ( $\nabla$ scaruso@unict.it)}

Gynecological Clinic of the Policlinico Universitario

\section{Antonio Cianci}

Gynecological Clinic of the Policlinico Universitario

Marco Iraci Sareri

Gynecological Clinic of the Policlinico Universitario

Marco Marzio Panella

Gynecological Clinic of the Policlinico Universitario

\section{Giuseppe Caruso}

University of Catania

\section{Stefano Cianci}

University of Messina

\section{Research Article}

Keywords: Hormonal contraceptives, Dienogest, Endometriosis-associated chronic pelvic pain, Nomegestrol acetate, Quality of life, Sexual activity, $17 \beta$-estradiol

Posted Date: February 25th, 2022

DOI: https://doi.org/10.21203/rs.3.rs-1323526/v1

License: (c) (1) This work is licensed under a Creative Commons Attribution 4.0 International License. Read Full License 


\section{Abstract}

Background: To evaluate the effects of a combined oral contraceptive containing $1.5 \mathrm{mg} 17 \mathrm{~b}$-estradiol (E2) and $2.5 \mathrm{mg}$ nomegestrol acetate (NOMAC) or $2 \mathrm{mg} /$ daily dienogest (DNG) oral progestin on endometriosis-associated chronic pelvic pain (CPP) and on the quality of life (QoL) and sexual function, by a randomized study design.

Methods: The E2/NOMAC group and DNG group included 99 and 98 women, respectively. The levels of CPP were measured by the visual analogic scale (VAS). The QoL scores were investigated by the Short Form-36 (SF-36). Finally, the sexual function was studied using the Female Sexual Function Index (FSFI), while the sexual distress by the Female Sexual Distress Scale (FSDS). The study had 3, 6 and 12-month follow-ups.

Results: The intra-group analysis showed an improvement of the VAS score from baseline to the 12month follow-up in women of both groups $(p<0.001)$. The inter-group comparison showed a similar improvement of CPP $(p=0.06)$. Women on DNG had better SF-36 somatic $(p<0.01)$ and FSFI scores $(p<0.006)$ than women on E2/NOMAC at 6 - and 12-month follow-ups.

Conclusions: The results support the efficacy of both hormonal treatments, even if DNG was more effective than E2/NOMAC in a limited intergroup comparison.

The research protocol was approved by the institutional review board of the Ethics Committee of the University Hospital Polyclinic, Catania, Italy, registered n. 109/2017/PO

\section{Introduction}

Chronic pelvic pain (CPP) due to endometriosis is a disabling symptom that affects about $10 \%$ of women of childbearing age. ${ }^{1}$ Rather than adopting extended or continuous treatments to control their CPP, women use on-demand non-steroidal anti-inflammatory drugs (NSAIDs), obtaining transient and poor symptom resolution. ${ }^{2}$ They begin to take hormonal therapies, usually combined hormonal contraceptives $(\mathrm{CHC})$ or progestins, when they are diagnosed with endometriosis by their doctors. ${ }^{3,4}$

Among the most recent $\mathrm{CHCs}$, a pill having $1.5 \mathrm{mg} 17 \beta$-estradiol (E2) and $2.5 \mathrm{mg}$ nomegestrol acetate (NOMAC) is used, with 24 days of active pill and 4-day of placebo pill regimen. The progestin activity of NOMAC is able to cover the 4-day hormone-free interval; this is due to the its long half-life of $45-50 \mathrm{~h}{ }^{5}$

Moreover, progestins are an alternative option for inhibiting estrogen-induced lesion proliferation and reducing endometriosis-associated CPP. Of the progestogens, Dienogest (DNG) $2 \mathrm{mg}$ per day is the only approved for the clinical treatment of endometriosis. ${ }^{6}$ The effects of DNG on endometriotic lesions are manifold; in fact, it has not only antiproliferative but also antiangiogenic and immunological activities. ${ }^{6} \mathrm{~A}$ peculiarity of the aforementioned hormonal treatments is that they are effective as long as they are used. In fact, when the woman stops their intake, the endometriosis-associated symptoms could reappear. 
The aim of the study was to evaluate the effects of E2/NOMAC or DNG on endometriosis-associated CPP (the primary endpoint) and on the quality of life (QoL) and sexual function (the secondary endpoints) of women, by a randomized study design.

\section{Methods}

According to the ESHRE guidelines, ${ }^{7} 347$ women aged $18-39$ years old (mean age $27.3 \pm 7.8$ ), affected by CPP, dysmenorrhea and dyspareunia have been recruited to participate in the study. Each woman had been using on-demand non-steroidal anti-inflammatory drugs (NSAIDs) from 15 months to 8 years.

Before enrollment, the inclusion and exclusion criteria for $\mathrm{CHC}$ or only-progestin usage were assessed medically. Furthermore, the exclusion criteria included women on GnRh or hormonal treatments within 6 or 3 months, respectively; or woman or her partner with sexual dysfunction; or not to have any sexual activity. All included women underwent physical and gynecological examinations, and finally transvaginal sonography (TVS). Consequently, 114 (32.8\%) women with clinical signs and TVT diagnosed with recto-vaginal endometriosis [44 (12.7\%)], ovarian endometrioma [49 (14.1\%)], or adenomyosis [21 (6\%)] were excluded. They were included in a surgical arm not considered in this study. Finally, 233 (67.2\%) women received counseling on the benefits of E2/NOMAC intake in an extended 24/4 regimen and of progestogen DNG 2 mg daily. However, after counseling 36 (15.5\%) women decided not to participate in the study, claiming to use NSAID on-demand therapy, while 197 (76.7\%) women accepted to participate in the study. According to a computer-generated list to randomize participants $1: 1$, each woman was allocated to the E2/NOMAC group or DNG group.

Ethics Committee Catania 1, University Hospital Polyclinic, Catania, Italy, approved the study protocol. It conformed to the ethical guidelines of the 2013 Helsinki Declaration. Informed written consent was obtained from each woman before entering the study. None of them received any payments. The time of enrollment was from October 2017 to November 2019.

\section{Instruments}

The Visual Analogic Scale (VAS) was used to define endometriosis-associated pain, ${ }^{8}$ nominally CPP, dysmenorrhea and dyspareunia. The Short Form-36 (SF-36) questionnaire was used to assess QoL. ${ }^{9}$ To assess sexual behavior the self-administered Female Sexual Function Index (FSFI) validated in the Italian gynecological population was assessed. ${ }^{10}$ An FSFI cut-off of $\leq 26.55$ is usually accepted for the diagnosis of sexual dysfunction in women within a wide age range. Moreover, for the diagnosis of sexual dysfunction, an essential element is the requirement that the condition causes significant personal distress for the woman. Therefore, the Female Sexual Distress Scale (FSDS) was used, ${ }^{11}$ having a cut-off of $\geq 15$.

The study included three follow-ups at 3, 6 and 12 months. All questionnaires were administered to both groups at the baseline evaluation and at each follow-up. 


\section{Statistical analysis}

Assuming a standard deviation of 4 and a mean difference of 3 with a $p \leq 0.05$ for the primary outcome measure (VAS), the sample size calculation indicated that 84 subjects would be the minimum number for each study arm required to have $95 \%$ statistically significant power.

Considering a dropout rate of $25-30 \%,{ }^{12} 196$ women were considered the number of subjects to be invited to participate in the study.

Intention-to-treat analyses were performed for all efficacy variables and included all women who had undergone the baseline evaluation and had at least one efficacy assessment after the baseline visit. The last observation carried forward method was used to select data such that missing data were replaced by values from the last available assessment during treatment before the respective assessment. The $\mathbb{\Xi}^{2}$ and ANOVA were used to compare the demographic and clinical data between the two groups, respectively. The difference was estimated with the $95 \%$ confidence interval $(\mathrm{Cl})$. Paired Student's test-test was used to compare the values obtained at baseline with those of the follow-ups from the SF-36 domains and VAS. For comparisons of the values obtained from the FSFI items between baseline and the follow-ups, the nonparametric Wilcoxon rank-sum test with $z$ values was used. Scores are presented as mean $\pm S D$. The correlation analyses with Pearson's $r$ coefficient were performed to examine the relationships between the VAS and FSFI scores. The result was statistically significant when $p<0.05$. Statistical analysis was carried out using the Primer of Biostatistics statistical computer package (Glantz SA, New York, USA: McGraw-Hill, Inc. 1997).

\section{Results}

Women were randomized to the E2/NOMAC group (n.99) or the DNG group (n.98). Table 1 shows the demographic characteristics of both groups at baseline. 
Table 1

Demographic characteristics

\begin{tabular}{|c|c|c|c|}
\hline & $\begin{array}{l}\text { E2/NOMAC group } \\
n=99\end{array}$ & $\begin{array}{l}\text { DNG group } \\
n=98\end{array}$ & $\mathbf{P}$ \\
\hline Age range (years) & 18 to 38 & 18 to 39 & 1 \\
\hline Mean Age & $26.4 \pm 6.8$ & $27.4 \pm 8.3$ & 0.3 \\
\hline $\mathrm{BMI} \mathrm{kg/m2}$ & $21.8 \pm 4.7$ & $22.1 \pm 3.5$ & 0.6 \\
\hline Age at menarche & $12.6 \pm 2.4$ & $12.8 \pm 3.1$ & 0.6 \\
\hline Menstrual cycle length (days) & 26 to 32 & 26 to 33 & 1 \\
\hline Duration of menses (days) & $4.5 \pm 2.2$ & $4.1 \pm 1.8$ & 0.1 \\
\hline Chronic Pelvic Pain & $99(100 \%)$ & $98(100 \%)$ & 1 \\
\hline Dysmenorrhea & $73(73.7 \%)$ & $74(75.5 \%)$ & 0.9 \\
\hline Dyspareunia & $62(62.6 \%)$ & $63(64.3 \%)$ & 1 \\
\hline Parity & $74(74.7 \%)$ & $75(76.5 \%)$ & 0.9 \\
\hline Nulliparous & $25(25.3 \%)$ & $23(23.5 \%)$ & 0.9 \\
\hline \multicolumn{4}{|l|}{ One or more children } \\
\hline Cigarette smoking & $84(84.8 \%)$ & $82(83.7 \%)$ & 0.9 \\
\hline Non-smoker & $15(15.2 \%)$ & $16(16.3 \%)$ & 0.7 \\
\hline Current smoker & $15.3 \pm 3.2$ & $14.7 \pm 4.2$ & 0.2 \\
\hline \multicolumn{4}{|l|}{ Daily cigarettes } \\
\hline Systolic blood pressure (mmHg) & $117.5 \pm 9.5$ & $119.2 \pm 5.7$ & 0.13 \\
\hline Diastolic blood pressure $(\mathrm{mmHg})$ & $71.3 \pm 7.8$ & $69.5 \pm 7.5$ & 0.1 \\
\hline Heart rate $(x \min )$ & $72.5 \pm 8.6$ & $70.6 \pm 5.5$ & 0.06 \\
\hline
\end{tabular}

At the 3-month follow-up, 10 (10.1\%) women of the E2/NOMAC group discontinued due to spotting; the dropout rate was $12.1 \%$ (twelve women) and $11.2 \%$ (eleven women) at the 6-and 12-month follow-up, respectively. Therefore, 66 (66.6\%) women completed the study. On the other hand, $6(6.2 \%)$ and 4 (4.1\%) women on DNG discontinued treatment due to spotting at the 3- and the 6-month follow-ups, respectively. At the 12-month follow-up, the dropout rate was $14.7 \%$ (thirteen women). Therefore, 75 (76.5\%) women completed the study. Intention-to-treat analyses were performed as reported in the statistical analysis. In addition, in their daily diary the women on E2/NOMAC recorded mild adverse events at the 3-month follow-up, which did not provoke discontinuation, namely spotting ( $16=17.9 \%)$, nausea $(12=135 \%)$, or breast tenderness $(13=14.6 \%)$. Moreover, some women on DNG reported mild adverse events at the 3- 
month follow-up, which did not provoke discontinuation, namely spotting $(17=18.4 \%)$, nausea (11 = $11.9 \%)$, or breast tenderness $(15=16.3 \%)$. Finally, $17(18.1 \%)$ and $20(30.3 \%)$ women on E2/NOMAC, and $19(20.6 \%)$ and 49 (65.3\%) women on DNG reported amenorrhea at the 3- and the 12-month follow-ups, respectively.

The intra-group analysis showed an improvement of the VAS score from baseline to the 12-month followup in women of both the E2/NOMAC and DNG groups $(p<0.001)$. The inter-group comparison showed no differences between baseline $(p=0.08)$, and the $3-(p=0.06)$ and 12-month $(p=0.06)$ follow-up values. On the contrary, at the 6-month follow-up women on DNG had a better improvement than women on E2/NOMAC ( $p=0.01)$ (Fig. 1).

Women of both groups had a significant reduction of dysmenorrhea and dyspareunia at each follow-up compared to baseline values $(p<0.001)$. No difference was observed by intragroup analysis at baseline $(p=0.2)$. At the 3-month follow-up, the DNG group had a better improvement than the E2/NOMAC group for both dysmenorrhea $(p=0.02)$ and dyspareunia $(p=0.003)$. Finally, at the 6 -and 12-month follow-ups the differences were not statistically significant ( $p \leq 0.2)$ (Fig. 2).

The QoL of the women of both groups improved from the 3-month to the 12-month follow-up $(p<0.001)$. The intergroup differences were not statistically significant at each follow-up for mental scores $(p \geq$ $0.16)$, and at baseline and the 3-month follow-up for the somatic score $(p \geq 0.18)$. Nevertheless, women on DNG had a better somatic score than women on E2/NOMAC at both the $6(p=0.01)$-and 12-month follow-ups $(p<0.006)$ (Fig. 3).

The FSFI and FSDS improved from the 3 to the 12 -month follow-ups in both groups $(p<0.001)$. The FSFI intergroup differences were not statistically significant at the 3-month follow-up $(p=0.15)$, but women on DNG had a better FSFI score than the women on E2/NOMAC at the $6(p=0.005)$ - and 12 -month followups $(p=0.006)($ Table $2 A)$.

On the other hand, the FSDS score of the women on DNG was better than that of the women on E2/NOMAC only at the 3-month follow-up $(p<0.001)$. In fact, at the $6-(p=0.68)$ and $12-(p=0.33$ month follow-ups there were no statistically significant intergroup differences (Table 2B). 
Table 2

(A) Female Sexual Function Index (FSFI) and (B) Female Sexual Distress Scale (FSDS) intragroup and intergroup comparison of women with endometriosis-associated pain symptoms at baseline and at 3, 6 and 12 months of $24 / 4$ regimen $17 \beta$-estradiol $(1.5 \mathrm{mg})$ and Nomegestrol Acetate $(2.5 \mathrm{mg})$ (E2/NOMAC) combined oral contraceptive, or of Dienogest (DNG) 2 mg daily.

\begin{tabular}{|c|c|c|c|c|c|}
\hline $\begin{array}{l}\text { A } \\
\text { FSFI score }\end{array}$ & Baseline & $\begin{array}{l}\text { 3rd month } \\
\text { follow-up }\end{array}$ & $\begin{array}{l}\text { 6th month } \\
\text { follow-up }\end{array}$ & $\begin{array}{l}\text { 12th month } \\
\text { follow-up }\end{array}$ & $p$ \\
\hline $\begin{array}{l}\text { E2/NOMAC } \\
\text { Group }\end{array}$ & $20.8 \pm 1.9$ & $27.5 \pm 1.6$ & $28.6 \pm 2.5$ & $30.2 \pm 2.8$ & $\dot{0}_{0.001}$ \\
\hline DNG Group & $21.1 \pm 1.2$ & $27.8 \pm 1.3$ & $29.7 \pm 2.9$ & $31.3 \pm 2.7$ & $\begin{array}{l}< \\
0.001\end{array}$ \\
\hline \multirow[t]{2}{*}{$p$} & 0.18 & 0.15 & 0.005 & 0.006 & \\
\hline & $\begin{array}{l}95 \% \mathrm{Cl},-0.7 \text { to } \\
-0.1\end{array}$ & $\begin{array}{l}95 \% \mathrm{Cl},-0.7 \text { to } \\
-0.1\end{array}$ & $\begin{array}{l}95 \% \mathrm{Cl},-1.9 \text { to } \\
-0.2\end{array}$ & $95 \% \mathrm{Cl},-2$ to -0.1 & \\
\hline $\begin{array}{l}\text { B } \\
\text { FSDS score }\end{array}$ & Baseline & $\begin{array}{l}\text { 3rd month } \\
\text { follow-up }\end{array}$ & $\begin{array}{l}\text { 6th month } \\
\text { follow-up }\end{array}$ & $\begin{array}{l}\text { 12th month } \\
\text { follow-up }\end{array}$ & $p$ \\
\hline $\begin{array}{l}\text { E2/NOMAC } \\
\text { Group }\end{array}$ & $18.5 \pm 1.5$ & $13.6 \pm 1.7$ & $10.2 \pm 1.8$ & $10 \pm 1.4$ & $\begin{array}{l}< \\
0.001\end{array}$ \\
\hline DNG Group & $18.4 \pm 1.3$ & $11.3 \pm 1.4$ & $10.1 \pm 1.6$ & $9.8 \pm 1.5$ & $\begin{array}{l}< \\
0.001\end{array}$ \\
\hline \multirow[t]{2}{*}{$p$} & 0.6 & $<0.001$ & 0.68 & 0.33 & \\
\hline & $\begin{array}{l}95 \% \mathrm{Cl},-0.3 \text { to } \\
-0.5\end{array}$ & $\begin{array}{l}95 \% \mathrm{Cl}, 1.8 \text { to } \\
-2.7\end{array}$ & $\begin{array}{l}95 \% \mathrm{Cl},-0.4 \text { to } \\
-0.6\end{array}$ & $\begin{array}{l}95 \% \mathrm{Cl},-0.2 \text { to }-0- \\
6\end{array}$ & \\
\hline
\end{tabular}

Moreover, the FSFI scores demonstrated a negative correlation with VAS values, more statistically significant in the E2/NOMAC group $(r-0.99 ; p<0.004)$ than in the DNG group $(r-0.96 ; p<0.03)$.

Finally, the intergroup satisfaction rate was similar at each follow-up. No woman of each group reported to be dissatisfied or very dissatisfied during the treatment.

\section{Discussion}

This randomized study had as the primary endpoint to investigate the efficacy of E2 / NOMAC or DNG intake in women with CPP associated with endometriosis-associated CPP, during 12 months of treatment. The design of the study was based on critical observation of the survey length. In fact, efficacy studies are usually limited to 3-6 months. By adopting such a design, we often cannot know what longer-term effects and benefits the treatment may have and how many people discontinue it. ${ }^{13}$

Although women of both groups had an improvement in their endometriosis-associated pain, from the 3month follow-up to the end of the study, women on DNG had a better improvement of their pain 
symptoms than those on E2/NOMAC.

However, the improvement of QoL and sexual life of both groups could be due to the progressive reduction of the pain syndrome reported by women. Moreover, the intergroup comparison showed a better improvement of QoL and sexual function in women who were using DNG than in those on E2/NOMAC, mainly for the somatic scores at the 6 - and 12-month follow-ups.

Today, several progestogens, although not approved to treat pain syndrome associated with endometriosis, are widely prescribed. ${ }^{3}$ DNG, unlike other progestogens, has been approved for medical treatment of endometriosis ${ }^{6}$, by continuous or extended regimens. ${ }^{14}$ DNG $2 \mathrm{mg}$ daily has been shown to have insufficient contraceptive activity ${ }^{15}$, therefore women who do not wish to become pregnant could use an estrogen-progestogen contraceptive. One of its catabolic characteristics consists in its half-life of $10 \mathrm{~h}$, in a continuous or extended regimen; therefore, it must be administered on a continuous or extended regimen, associated or combined with an estrogen. Treatment of endometriosis-associated pain symptoms should exclude hormone-free interval regimens. In fact, if this were the regimen, women may complain of symptoms returning during the hormone-free interval. Authors showed that DNG combined with estradiol valerate in a $26 / 2$ four-phase association was effective on the pain syndrome. ${ }^{16}$ The proliferative activity of the endometrial epithelium induced by estrogen is well known, as well as the inhibitory activity of progesterone towards its proliferation. The synchronous activity of the two steroids in ectopic endometrial tissue is not respected. In fact, the unbalanced activity between the two steroids, with estrogen predominance, promotes a chronic inflammatory state. The inability of progesterone to balance the proliferative activity of estrogen depends on altered or reduced of its receptor expression in endometriosis tissues. ${ }^{17}$ Reduced progesterone activity is accompanied by low levels of $17 \beta$ hydroxysteroid dehydrogenase (17 $\beta-H S D)$ type 2 production, whereby E2 is not converted to estrone, its biologically less potent metabolite; moreover, Furthermore, in endometriosis tissue, there is an anarchic production of p450 aromatase expression which increases E2 level. ${ }^{18}$ Increased neuroinflammation and neoangiogenesis due to increased E2 supports the persistence of the CPP. ${ }^{19}$ Because of what has been mentioned above, when E2 is used in hormonal contraception, it could have metabolic and tissue effects that are less than those of EE, Indeed, it is interesting to know that $5 \mathrm{mg}$ of EE is equivalent to about $1 \mathrm{mg}$ E2. Therefore, the common usage of low-dose OCs containing 20 to $30 \mathrm{mg}$ of EE is equivalent to 4 to 6 times the physiologic dose of E2. ${ }^{20}$

The main therapeutic activity of progestogens in endometriosis is due to progesterone receptor signaling, which induces downregulation of estrogen receptors. ${ }^{21}$ Dienogest is able to inhibit aromatase expression and, as a result, local estrogen production is reduced..$^{22}$ Moreover, Dienogest inhibits the expression of $17 \beta-H S D$ type 1 , the enzyme that catalyzes the reduction of estrone to estradiol, and upregulates the expression of the oxidative $17 \beta-$ HSD type 2 , which inactivates estradiol. ${ }^{23}$

NOMAC is a 19-norprogesterone derivative that binds specifically to the progesterone receptor; it has strong antiestrogenic effects, inhibiting $17 \beta-H S D$ type 1 , with a consequent reduction of conversion of 
estrone to estradiol. ${ }^{24}$ Moreover, it reaches a peak serum concentration within $4 \mathrm{~h}$ after oral administration and, unlike DNG, its half-life is approximately $50 \mathrm{~h} .{ }^{5}$ Thus, in the 24/4 E2/NOMAC OC regimen, the steroidal activity of NOMAC is able to cover the 4-day hormone-free interval. In fact, women on E2/NOMAC usually have reduced bleeding, and some women experience amenorrhea. ${ }^{25}$ In our study $30.3 \%$ of women had amenorrhea, however, less than the women on DNG (65.3\%). Reduction in bleeding or even more so the amenorrhea observed in both groups could decrease or disrupt, respectively, the retrograde flow of menstrual tissue through the fallopian tubes, that is one of the most established hypotheses of endometriosis pathogenesis. ${ }^{26}$

It is important to consider that CPP and dysmenorrhea usually start during adolescence, but treatments are often started several years later and are not always adequate to limit or reduce the progression of endometriosis. Early diagnosis and treatment are essential in order to decrease neoangiogenesis and neuroinflammation and so the chronic inflammatory status, and preserve future fertility. ${ }^{27}$ Pain symptoms usually reappear when estrogen-progestin or only progestin intake is discontinued. In fact, progestins and combined hormonal contraceptives do not eliminate endometriotic lesions but induce their quiescence. ${ }^{28}$ Consequently, their usage has to be for a long time. However, unlike progestin-only contraceptives, biological data support the results that prolonged use of estrogen-progestogen contraception could promote a progression of endometriotic lesions. Consequently, some authors support the use of progestin-only contraceptives rather than OCs, considering them as first-line therapy for treating endometriosis CPP. ${ }^{29}$

In all the women using E2/NOMAC or DNG, QoL and sexual function improved. Even if receptor binding studies showed that DNG has approximately one-third of the anti-androgenic activity of cyproterone acetate, and could affect the libido of long-term users, recent studies showed that the quality of sexual life, particularly, improves during DNG treatment of women with endometriosis. ${ }^{13}$ On the other hand, women using E2/NOMAC had benefits on their sexual function and sexual distress. ${ }^{30}$ In fact, E2 could have less antiandrogenic activity than EE by inducing less SHBG improvement. ${ }^{31}$ In addition to the effects due to the biological activity of steroids, the reduction of painful symptoms could favor subjective well-being, such as improving the quality of sexual life. In fact, although DNG has an antiandrogenic activity that could reduce sexual desire and arousal, the decrease in painful symptoms, especially dyspareunia, may be capable of promoting satisfactory sexual function. ${ }^{13}$

\section{Conclusion}

Our study was based on the clinical diagnostic symptoms of CPP associated with endometriosis, and not on the laparoscopic diagnosis, which remains the gold standard. This could be a limit of our study. However, today, laparoscopy is a very limited investigation for the diagnosis of endometriosis, and hormone therapy is initiated based on painful symptoms reported by the woman. Nevertheless, laparoscopic diagnosis to confirm endometriosis could be taken into consideration in future full studies. 
The design of our study did not consider the contraceptive needs of the women enrolled, as it was a randomized study. In fact, each woman was advised that DNG should not be considered as a contraceptive. On the other hand, the women enrolled had no contraceptive needs. Moreover, we need to expand the number of investigations using E2/Progestogen rather than EE/Progestogen contraceptives to understand if the effectiveness of the former is better or equal to that of the latter.

\section{Declarations}

\section{Ethics approval and consent to participate:}

Ethics Committee Catania 1, University Hospital Polyclinic, Catania, Italy, approved the study protocol. It conformed to the ethical guidelines of the 2013 Helsinki Declaration. Informed consent was obtained directly from all women who were over 18 years of age. Informed consent from parents was obtained for teenagers under 18.

Consent for publication: Not applicable

Availability of data and materials: the datasets generated and analyzed during the current study are not publicly available due to maintain the privacy of participants, but are available from the corresponding author upon reasonable request and with permission of the Ethics Committee.

Competing interests: The authors declare that they have no competing interests

Funding: none

\section{Authorship:}

Conception and Design: All authors

Acquisition of Data: SC; MIS; MMP; GC

Analysis and Interpretation of Data: SC; AC; MIS

Drafting the Article: SC; GC; StC

Revising It for Intellectual Content: All authors

Final Approval of the Completed Article: All authors

\section{Acknowledgments}

The authors wish to thank The Scientific Bureau of the University of Catania for language support.

\section{References}


1. Rogers PA, D'Hooghe TM, Fazleabas A, Gargett CE, Giudice LC, et al. Priorities for endometriosis research: recommendations from an international consensus workshop. Reprod Sci. 2009;16:335346

2. Marjoribanks J, Ayeleke RO, Farquhar C, Proctor M. Nonsteroidal anti-inflammatory drugs for dysmenorrhea. Cochrane Database Syst Rev. 2015;30;(7):CD001751.

3. Bishop LA. Management of Chronic Pelvic Pain. Clin Obstet Gynecol. 2017;60:524-530

4. Vercellini P, Frontino G, De Giorgi O, Pietropaolo G, Pasin R. Continuous use of an oral contraceptive for endometriosis-associated recurrent dysmenorrhea that does not respond to a cyclic pill regimen. Fertil Steril. 2003;80:560-563.

5. Lello S. Nomegestrol acetate: pharmacology, safety profile and therapeutic efficacy. Drugs 2010;70:541-559.

6. Harada T, Taniguchi F. Dienogest: a new therapeutic agent for the treatment of endometriosis. Womens Health (Lond Engl) 2010;6:27-35

7. Dunselman GAJ, Vermeulen N, Becker C, et al. European Society of Human Reproduction and Embryology. ESHRE guideline: management of women with endometriosis. Hum Reprod. 2014;29:400-412

8. Gallagher EJ, Liebman M, Bijur PE. Prospective validation of clinically important changes in pain severity measured on a visual analog scale. Ann Emerg Med. 2001;38:633-638

9. Bech P, Brazier J, Bullinger M, et al. The factor structure of the SF-36 Health Survey in 10 countries: Results from the International Quality of Life Assessment (IQOLA) project. J Clin Epidemiol. 1998;51:1159-1165

10. Nappi RE, Albani F, Vaccaro $P$, et al. Use of the Italian translation of the Female Sexual Function Index (FSFI) in routine gynecological practice. Gynecol Endocrinol. 2008;24:214-219

11. Derogatis LR, Rosen R, Leiblum S, Burnett A, Heiman J. The Female Sexual Distress Scale (FSDS): Initial validation of a standardized scale for assessment of sexually related personal in distress women. J Sex Marital Ther. 2002;28:317-330.

12. Caruso S, Cianci S, Vitale SG, Fava V, Cutello S, Cianci A. Sexual function and quality of life of women adopting the levonorgestrel-releasing intrauterine system (LNG-IUS $13.5 \mathrm{mg}$ ) after abortion for unintended pregnancy. Eur J Contracept Reprod Health Care. 2018;23:24-32.

13. Caruso S, Iraci M, Cianci S, Vitale SG, Fava V, Cianci A. Effects of long-term treatment with Dienogest on the quality of life and sexual function of women affected by endometriosis-associated pelvic pain. J Pain Res. 2019;12:2371-2378.

14. Schindler AE, Campagnoli C, Druckmann R, et al. Classification and pharmacology of progestins. Maturitas 2008;61:171-180.

15. Klipping C, Duijkers I, Remmers A, et al. Ovulation-inhibiting effects of dienogest in a randomized, dose-controlled pharmacodynamic trial of healthy women. J Clin Pharmacol.2012;52:1704-1713 
16. Grandi G, Xholli A, Napolitano A, Palma F, Cagnacci A. Pelvic pain and quality of life of women with endometriosis during quadriphasic estradiol valerate/dienogest oral contraceptive: a patientpreference prospective 24-week pilot study. Reprod Sci. 2015;22:626-632

17. Marquardt RM, Kim TH, Shin JH, Jeong JW. Progesterone and Estrogen Signaling in the Endometrium: What Goes Wrong in Endometriosis? Int J Mol Sci. 2019;20 pii: E3822.

18. Zeitoun K, Takayama K, Sasano H, et al. Deficient 17beta-hydroxysteroid dehydrogenase type 2 expression in endometriosis: Failure to metabolize 17beta-estradiol. J Clin Endocrinol Metab. 1998;83;4474-4480.

19. Morotti M, Vincent K, Becker CM. Mechanisms of pain in endometriosis. Eur J Obstet Gynecol Reprod Biol. 2017;209:8-13

20. Speroff L, Symons J, Kempfert N, Rowan J; femhrt Study Investigators. The effect of varying lowdose combinations of norethindrone acetate and ethinyl estradiol (femhrt) on the frequency and intensity of vasomotor symptoms. Menopause. 2000;7:383-390

21. Brichant G, Nervo P, Albert A, Munaut C, Foidart JM, Nisolle M. Heterogeneity of estrogen receptor a and progesterone receptor distribution in lesions of deep infiltrating endometriosis of untreated women or during exposure to various hormonal treatments. Gynecol Endocrinol. 2018;34:651-655.

22. Shimizu Y, Mita S, Takeuchi T, Notsu T, Mizuguchi K, Kyo S. Dienogest, a synthetic progestin, inhibits prostaglandin E2 production and aromatase expression by human endometrial epithelial cells in a spheroid culture system. Steroids. 2011;76:60-67.

23. Mori T, Ito F, Matsushima H, Takaoka O, Koshiba A, Tanaka Y, Kusuki I, Kitawaki J. Dienogest reduces HSD17ß1 expression and activity in endometriosis. J Endocrinol. 2015;225:69-76).

24. Shields-Botella J, Chetrite G, Meschi S, Pasqualini JR. Effect of nomegestrol acetate on estrogen biosynthesis and transformation in MCF-7 and T47-D breast cancer cells. J Steroid Biochem Mol Biol. 2005; 93:1-13.

25. Gerrits MG, Schnabel PG, Post TM, Peeters PA. Pharmacokinetic profile of nomegestrolacetate and $17 \beta$-estradiol after multiple and single dosing in healthy women. Contraception 2013;87:193-200.

26. Bulun SE, Yilmaz BD, Sison C, et al. Endometriosis. Endocr Rev. 2019;40:1048-1079

27. Clemenza S, Vannuccini S, Capezzuoli T, Meleca Cl, Pampaloni F, Petraglia F. Is primary dysmenorrhea a precursor of future endometriosis development? Gynecol Endocrinol. 2021;37:287293

28. Liang B, Wu L, Xu H, Cheung CW, Fung WY, Wong SW, et al. Efficacy, safety and recurrence of new progestins and selective progesterone receptor modulator for the treatment of endometriosis: a comparison study in mice. Reprod Biol Endocrinol. 2018;16:32.

29. Casper RF. Progestin-only pills may be a better first-line treatment for endometriosis than combined estrogen-progestin contraceptive pills. Fertil Steril. 2017;107:533-536.

30. Caruso S, Cianci A, Iraci M, Fava V, Di Pasqua S, Cianci S. Does Nomegestrol Acetate Plus 17ßEstradiol Oral Contraceptive Improve Endometriosis-Associated Chronic Pelvic Pain in Women? J Women's Health (Larchmt) 2020;29:1184-1191 
31. Caruso S, Agnello C, Romano M, Cianci S, Lo Presti L, Malandrino C, Cianci A. Preliminary study on the effect of four-phasic estradiol valerate and dienogest (E2V/DNG) oral contraceptive on the quality of sexual life. J Sex Med. 2011;8:2841-2845.

\section{Figures}

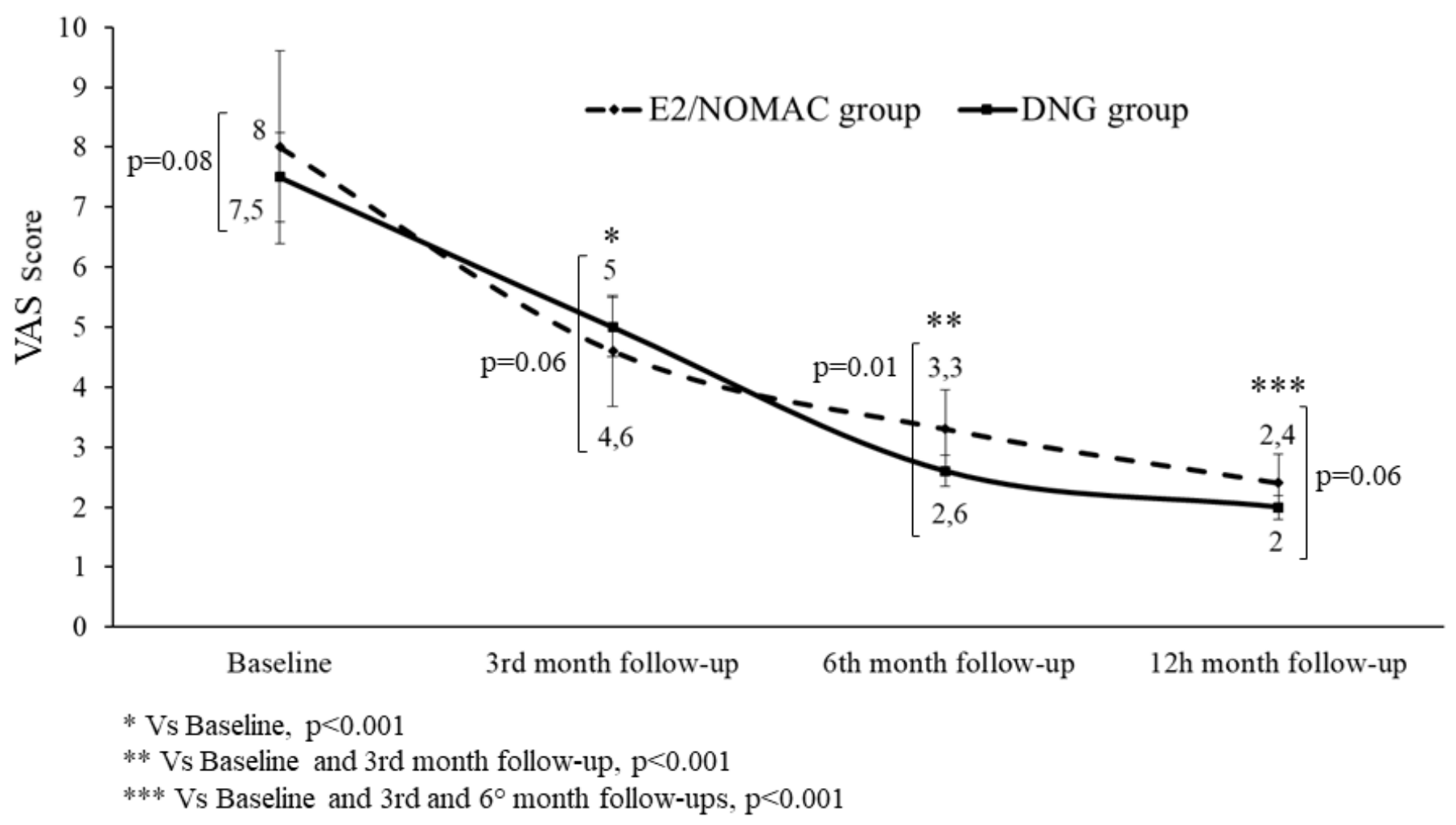

\section{Figure 1}

Visual Analog Scale (VAS) score of women affected by endometriosis-associated chronic pelvic pain at 3, 6 and 12 months of $24 / 4$ regimen $17 \beta$-estradiol $(1.5 \mathrm{mg})$ and Nomegestrol Acetate (2.5mg) (E2/NOMAC) combined oral contraceptive, or of Dienogest (DNG) $2 \mathrm{mg}$ daily. 


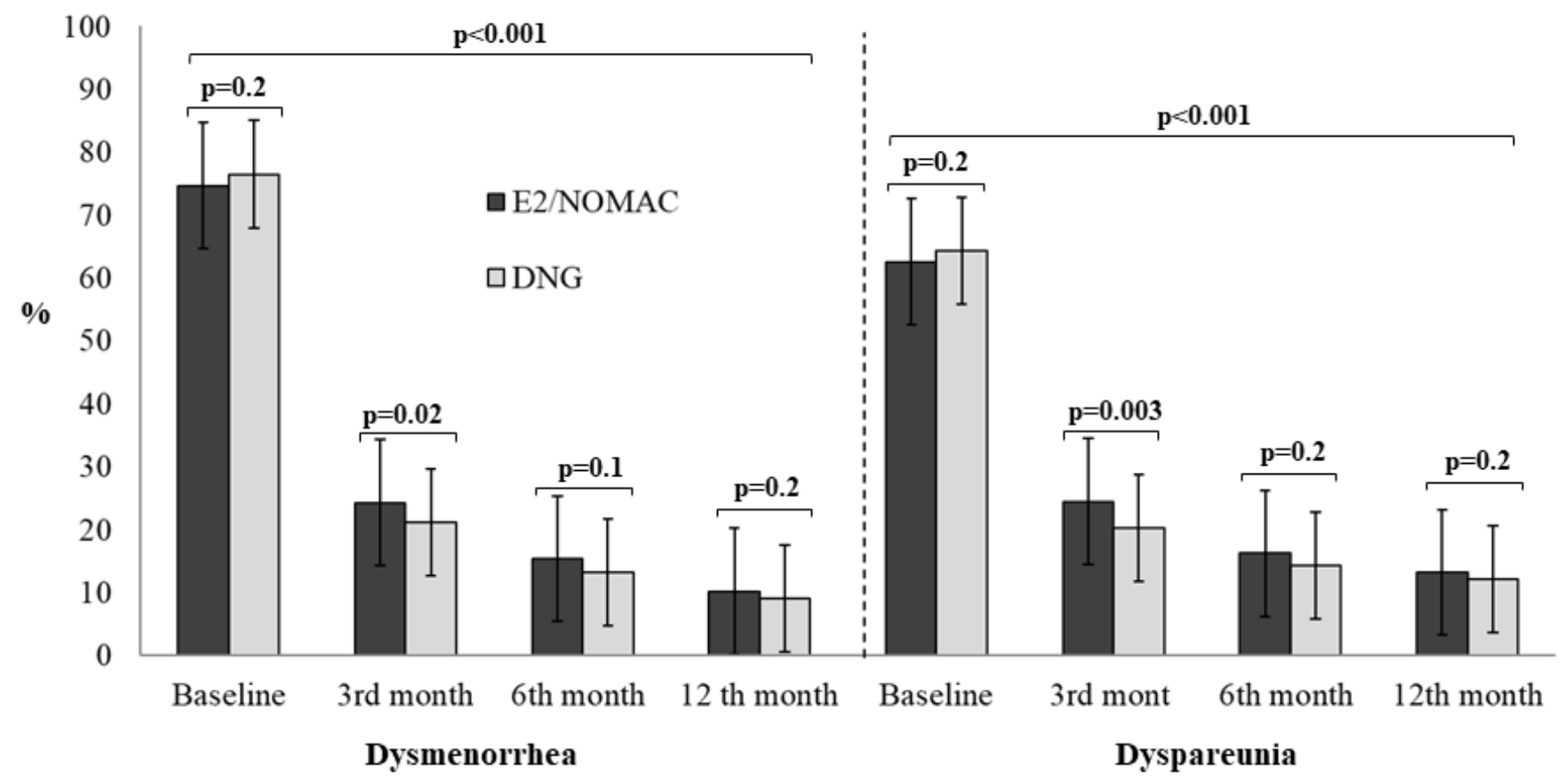

Figure 2

Dysmenorrhea and dyspareunia intragroup and intergroup comparison of women with endometriosisassociated pain symptoms at baseline and at 3,6 and 12 months of $24 / 4$ regimen $17 \beta$-estradiol (1.5mg) and Nomegestrol Acetate (2.5mg) (E2/NOMAC) combined oral contraceptive, or of Dienogest (DNG) $2 \mathrm{mg}$ daily. 


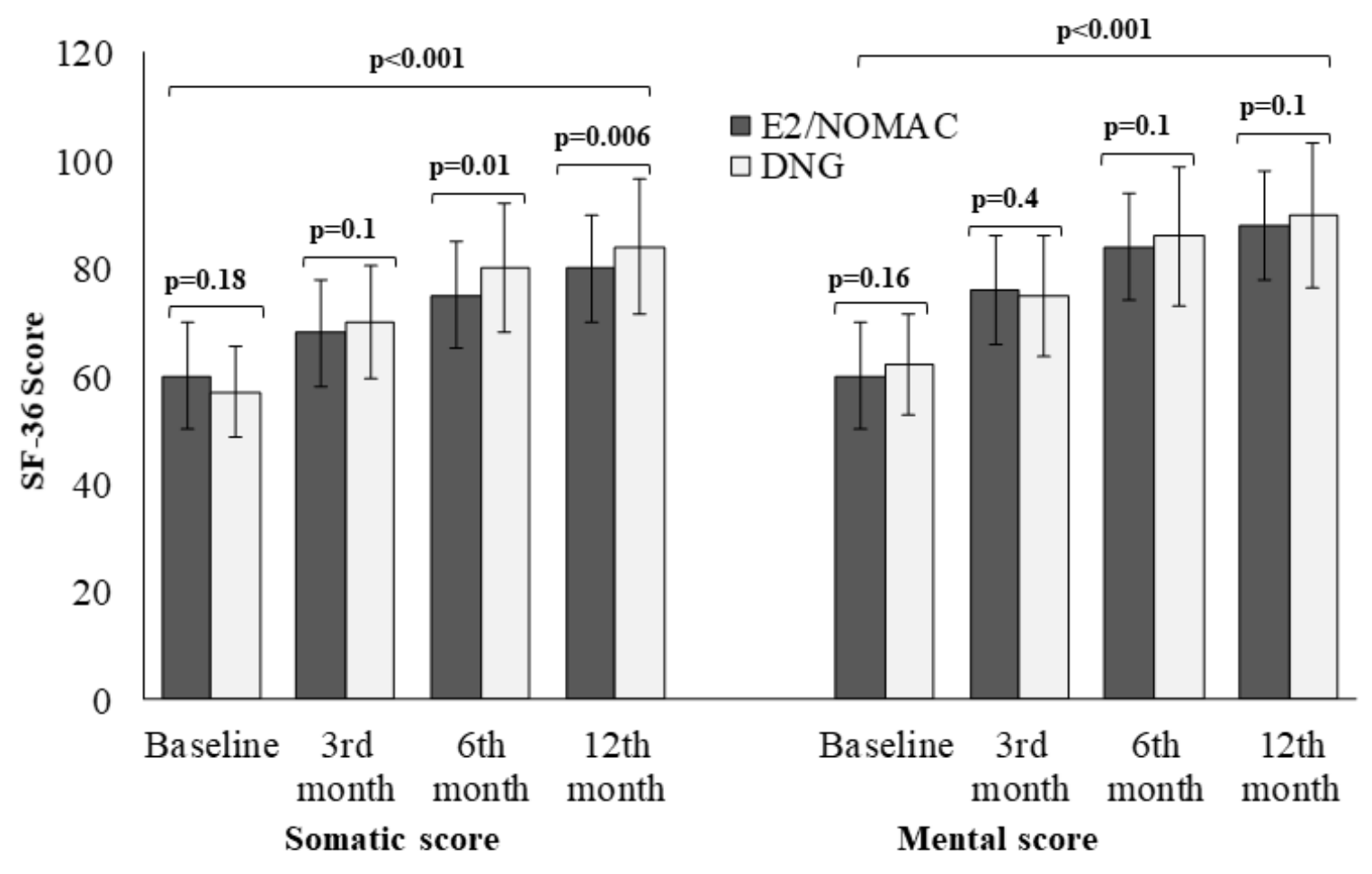

Figure 3

Quality of Life intragroup and intergroup comparison of women with endometriosis-associated pain symptoms at baseline and at 3,6 and 12 months of $24 / 4$ regimen $17 \beta$-estradiol $(1.5 \mathrm{mg})$ and Nomegestrol Acetate (2.5mg) (E2/NOMAC) combined oral contraceptive, or of Dienogest (DNG) $2 \mathrm{mg}$ daily. 